\title{
Kinematic and Dynamic Simulation Analysis of Hydraulic Excavator's Working Equipment based on ADAMS
}

\author{
Hong Yan YU ${ }^{1}$, Ming Hai YUAN ${ }^{1,2, a}$, Kun DENG ${ }^{1}$ and Rong DONG ${ }^{1}$ \\ ${ }^{1}$ College of Mechanical and Electrical Engineering, Hohai University, Changzhou 213022, China \\ ${ }^{2}$ Guangxi Key Laboratory for Manufacturing System and Advanced Manufacturing Technology, Nanning, 530004, China
}

\begin{abstract}
This paper establishes the 3D excavator model according to the actual size in UG firstly. Then based on the virtual simulation software ADAMS, the virtual prototype of the working device is built by adding interrelated constraints(kinematic pair and hydraulic cylinder driving function) and load secondly. This paper gets the main parameters of the excavator working scope and the pressure situation change curves of point of each hydraulic cylinder by making kinematic and dynamic simulation analysis of hydraulic excavator's working equipment at last. The conclusion providing design theory and improvement for the excavator's working device, which also play an important role in improving the level of China's excavator design, enhancing excavator's performance and promoting the rapid development of excavator industry.
\end{abstract}

Key words: Hydraulic excavator, Kinematic simulation, Dynamic simulation, ADAMS

\section{Introduction}

As the main mechanical equipment of earthwork excavation, hydraulic excavator is widely used in the construction of water conservancy and hydropower engineering, building engineering, municipal engineering and other engineering with the advantages of great digging force, stable transmission, simple manipulation and so on. And the excavator's working equipment plays an important part to make sure hydraulic excavator can complete all the functions. Under the actual working condition, the reliable performance and the working performance of the excavator is directly decided by the motion trajectory and the stress situation. Therefore, it is very important to design and study the working device of excavator. The traditional design method can not only cause the repetitive waste of manpower and material resources, but also result in some kinetic parameters are inconsistent with the design in manufactured products due to the dynamics of virtual model experiment was not carried out in time.

Scholars in and abroad did lots of research on the excavator's working equipment. For example, DiMaio S.P $\mathrm{P}^{[1]}$ ect. established a simulator with human-computer interaction interface. It simulated the interaction force between the excavator working device and the ground, and simulated the excavator's machine, hydraulic system successfully. Haga.M ${ }^{[2]}$ ect. studied automatic mining control system, by controlling the horizontal motion of boom and bucket tooth to automatically control the mining depth. This method overcomes the problem that

\footnotetext{
${ }^{\text {a }}$ Corresponding author: ymhai@126.com
} 
operators repeat to determine the mining depth, so that the mining efficiency is significantly improved. Imanishi, Etsujiro $^{[3]}$ ect. obtained some deformation characteristics of the excavator working device through dynamic simulation of working process, which also verify the superiority of the dynamics simulation analysis method in excavator structure design and machine performance analysis test. Xianghong $\mathrm{Xu}^{[4]}$ ect. applied the grid computing technology to the finite element analysis, taking the excavator working device as an example, the optimization design was carried out. Caijun Xue ${ }^{[5]}$ ect. carried on the static and dynamic analysis of the excavator working device by using the method of the finite element analysis, combined with the stress test, verified the validity of the finite element analysis results.

At present, the domestic and foreign research for excavator working equipment mainly concentrated in the finite element analysis, motion analysis and optimization of mining device, the optimization methods are mostly carried out by programming, and the research in the overall mining force optimization and operation stability is less, for the study in multi components and multi software of working device is relatively small too, this leads to the research data too independent, the lack of correlation, unable to thoroughly solve the problem of the engineering practice. For this reason, this paper is about the mechanical simulation analysis for the kinematics and dynamic of hydraulic excavator's working equipment.

\section{The establishment of simulation model in ADAMS}

The UG model can be imported into ADAMS, when a certain working environment which can simulate the real environment (including setting the unit, setting the gravity, setting the working grid, etc.) is given. The model is shown in Fig.1. After import, first to add constraints and driving function, such as adding fixed pair between the base and the ground, adding rotating pair, column pair and ball joint pair at each part of the connection, adding mobile pair between each hydraulic cylinder and its corresponding piston rod.second to define the driving function of the kinematic constraint as the step function (i.e. STEP function). After above, last to check the model with the model checking tool. Ensuring the model has 0 degrees of freedom and 0 redundant constraints $^{[6]}$.

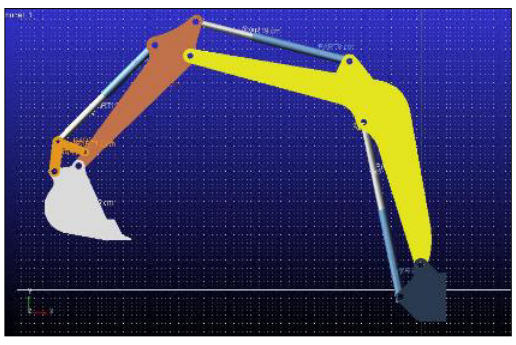

Fig.1 After the introduction of ADAMS model

\section{Kinematic simulation analysis}

\subsection{Motion parameters of Hydraulic cylinder working device}

Backhoe device geometry position is determined by the lift arm hydraulic cylinder length L1, bucket rod hydraulic cylinder length L2 and bucket hydraulic cylinder length L3. Obviously, The anti shovel device will be a certain position when L1, L2, and L3 are fixed ${ }^{[7]}$. Table 1 is the movement parameters of the hydraulic cylinder for the anti shovel hydraulic cylinder. 
Table 1. Movement parameters of $\mathrm{HC}$ working device

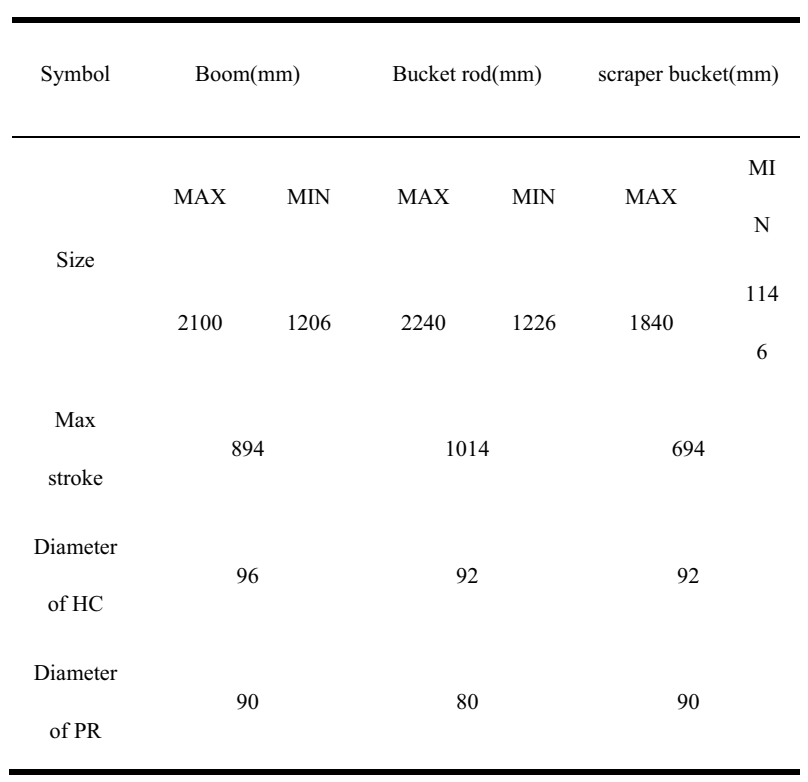

\subsection{Hydraulic cylinder motion function and motion trajectory simulation}

The Motion simulation of boom hydraulic cylinder, bucket rod hydraulic cylinder and bucket hydraulic cylinder are shown as follow:

\subsubsection{Motion simulation of boom hydraulic cylinder}

Condition: Adjust bucket rod cylinder in the full shrink state, motion function: step(time, $0,0,5,400)$. Adjust the bucket cylinder in the full shrink state, motion function:step(time, 5,0,10,452).

Movement function of boom hydraulic cylinder is step(time, 10,0,20,-154)+step(time,20,0,40,154+740).

Transfer ADAMS/Post processor Modular, to replay simulation results and draw curves, Tracking the moving track of the mark points of the bucket tooth of excavator in Trace Marker, trajectory chart of the mark point is shown in fig. 2 .

\subsubsection{Motion simulation of bucket rod hydraulic cylinder}

Condition: Adjust the boom cylinder in the full shrink state,motion function: step(time,0,0,15,740).Adjusting the connecting point between the boom and the bucket rod,the connecting point between the bucket rod and bucket, the bucket tooth being a straight line,

Motion function: step(time, 15,0,20,405).

Movement function of bucket hydraulic cylinder is step(time,20,0,35,-413)+step(time, 35,0,50,413+400).

Transfer ADAMS/Post processor Modular,to replay simulation results and draw curves, Tracking the moving track of the mark points of the bucket tooth of excavator in Trace Marker, trajectory chart of the mark point is shown in fig.3.

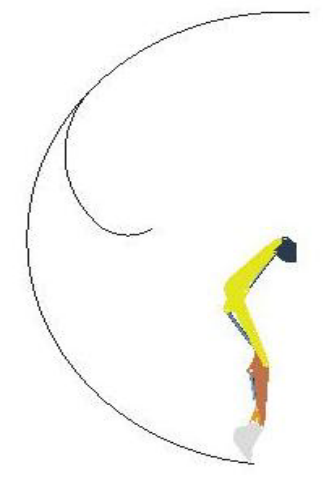

Fig.2 Moving arm simulation

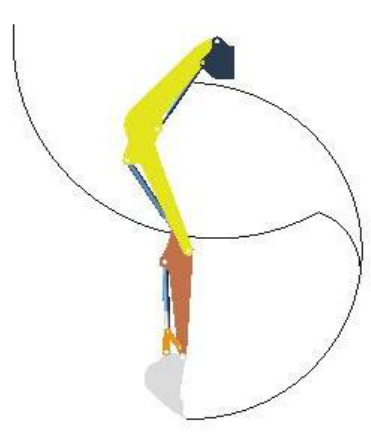

Fig.3 Bucket rod simulation

\subsubsection{Motion simulation of bucket hydraulic cylinder}

Excavator in mining only conducted by bucket hydraulic cylinder, the excavating trajectory is a circular arc, the arc line with the bucket and the bucket rod hinge point as the center, with the distance between the joint to the bucket tooth as the radius, and the wrap angle (i.e. the rotation angle of the bucket) and arc length is determined by the bucket hydraulic cylinder's stroke ${ }^{[8]}$.

\section{Dynamic simulation analysis}

Dynamic Simulation is to study the role of external loads of the components and systems of internal power relations changes, for excavator, the main research is to study the variation force of bucket tooth and hinge point. The purpose is to ensure the effectiveness of the design ${ }^{[9]}$.

\subsection{Load Calculation in complex mining process}

Soil resistance and friction are ignored. When the excavator working device is mining, the main external loads put on it are as follows. 


\subsubsection{Tangential and normal mining resistance}

Generally speaking, the tangential and normal mining resistance can be considered acting on the top of bucket tooth approximately, and the directions are the tangential and normal direction of mining track. Their empirical formula are as follows:

$$
\begin{aligned}
& W_{1}=K_{0} b h \\
& W_{2}=\psi W_{1}
\end{aligned}
$$

The $\mathrm{k}_{0}$ is mining ratio resistance, it reflects the total resistance on bucket-type excavation devices when it is mining. The resistance exists in soil failure, loading soil and friction. $\mathrm{k}_{0}$ 's unit is $N / \mathrm{cm}^{2}$.Small excavators usually work in III level soil. According to look-up table, $\mathrm{k}_{0}=15 \mathrm{~N} / \mathrm{cm}^{2}$.b-Cutting width, it's unit is $\mathrm{cm}$. According to the model, $b=60 \mathrm{~cm}$. h-Cutting depth, it'sunit is $\mathrm{cm}$. Generally, $\mathrm{h}=0.2 \mathrm{~b}=12 \mathrm{~cm}$. $\Psi$-Coefficient of Mining resistance. Look-up table shows, $\Psi=0.5$. By substituting the above values into the formula(1), (2)and calculating, we get following data. $\mathrm{W}_{1}=10800 \mathrm{~N}=10.8 \mathrm{KN}$, $\mathrm{W}_{2}=0.5 \mathrm{~W}_{1}=5.4 \mathrm{KN}$.

\subsubsection{Hoisting resistance}

Hoisting resistance mainly refers to the gravity of the materials in the bucket when excavation finished. As usual, it is added to the centroid of bucket, and it's direction is always vertical downward.

$$
\text { Gravity formula: } \quad G=\rho V g=\gamma V
$$

V-Bucket capacity. According to the design value, $\mathrm{V}=0.28 \mathrm{~m}^{3}$. $\rho$-Soil density. Density of III level soil is $1.8 \times 10^{4} \mathrm{~kg} / \mathrm{m}^{3}$. On the basis of gravity set in ADAMS, $\mathrm{g}=9.8 \mathrm{~m} / \mathrm{s}^{2}$. Then we can get the gravity value of the materials. It is about $5 \mathrm{KN}$.

\subsection{Add the load and drive in ADMAS}

The direction of tangential and normal resistance correspond to the the tangent and normal direction of the bucket tooth. It shows that these there forces are in one direction $^{[10]}$

By using common complex motion in digging as a work circle, we can determine the changes of force of each cylinder and hinge point when it works in the deepest position. Furthermore, we can get the kinetic parameters of excavation. Without considering the turn-around time, working cycle time including excavation time and unloading time. The excavation time usually depends on the speed of bucket digging. In order to facilitate the simulation, running speed values $0.5 \mathrm{~m} / \mathrm{s}$, the digging speed values $0.75 \mathrm{~m} / \mathrm{s}$, unloading time values $2 \mathrm{~s}$.

Based on the above analysis, we divide one cycle into three steps, and set the total cycle time as $8 \mathrm{~s}$ :

(1) Bucket downward phase(0s-3s): We adjust working device's digging position to maximum digging radius which means that shrinking the bucket rod and boom cylinder. In this process, we assume there are no external loads applied.

(2) Digging phase(3s-5s): Bucket rod and bucket work together until the bucket filled with soil. It means the completion of the digging operation. In this process, the load is the tangential and normal digging resistance. They increase with bucket angle increases from the beginning. When digging into the deepest, digging resistance reaches the maximum, then decrease with the increase of the bucket angle.

(3) Hoisting and unloading phase(5s-8s): Hoisting phase, first we adjust the boom cylinder to the full shrinking state, at the same time adjust the bucket cylinder to ensure objects not scattered in the process of ascension in the bucket. then adjust bucket rod and bucket cylinder to the full shrinking state, and complete unloading,and complete unloading. In mining process, main load is the gravity of the material, the gravity of the material along with the increase of material, and boom lifting stage stays the same.Three load function curves are as shown in fig. 4. 


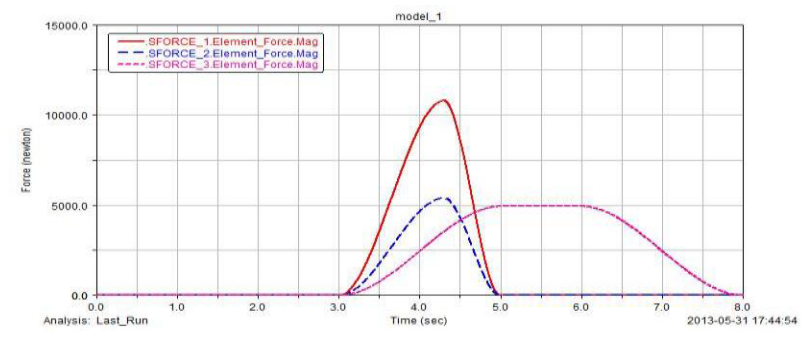

Fig.4 The change curve of the outside load

\subsection{Dynamic simulation analysis}

From motion simulation get the change of the connecting pointI between the boom and the bucket rod, the change of the connecting point $\mathrm{K}$ between the rocker rod and thebucket rod, the change of the connecting point $\mathrm{M}$ between the bucket rod and the bucket. the change of the connecting point $\mathrm{N}$ between the connecting rod and the bucket as shown in fig.5, The change of three hydraulic cylinder force as shown in fig.6: The figure shows that in the bucket down stage, each hinged point in 0-2.5 $\mathrm{s}$ basic is not affected by external forces, so the force variation is small, Lower movable arm due to mainly complete arm action, so the torque change largely as relative. In $2.5 \mathrm{~s}$ to $3.5 \mathrm{~s}$ mining process, the bucket tooth withstand from tangential and normal resistance, so the force changes in the rising trend. Mining process is mainly the bucket rod and the role of bucket hydraulic cylinder, relatively, hinge points I and $\mathrm{M}$ stress is larger; Mining end improve unloading phase $3.5 \mathrm{~s}-6 \mathrm{~s}$, which in ascending within 3.5 $\mathrm{s}$ to $4.5 \mathrm{~s}$, movable arm plays a main role, but relative to the digging force is small lot, other hinge point more smoothly. In the unloading process of $5 \mathrm{~s}-6 \mathrm{~s}$, in order to be able to unload thoroughly, they are usually based on a great speed in a short period of time, this leads to the stress of the hinged points of ups and downs is bigger. Seeing from the diagram, there is an increase to reduce, the change of this force in the process is consistent with the actual working conditions. The result can be used as a reference, the subsequent design calculation and the change of three hydraulic cylinder force which can be taken as reference to the machine hydraulic joint simulation.

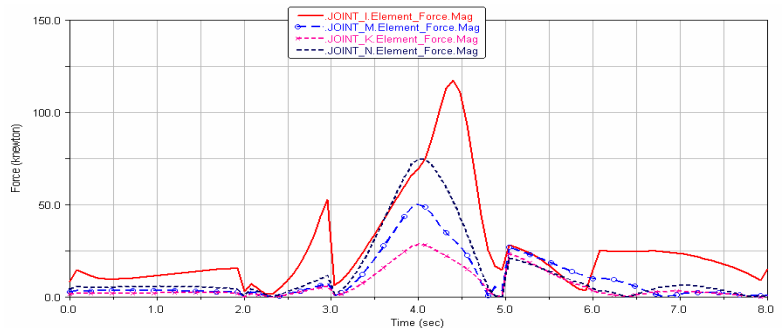

Fig. 5 Articulated point force curve

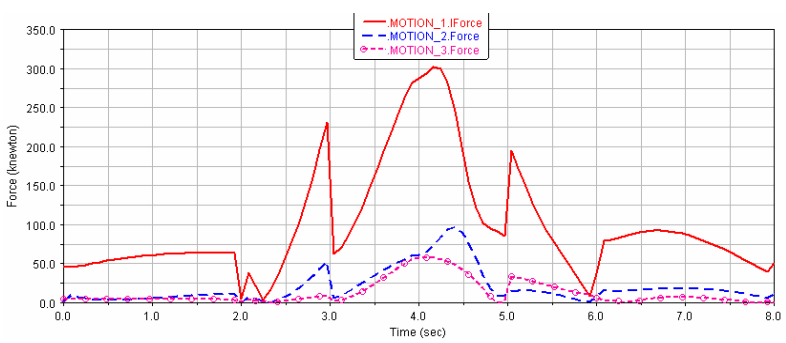

Fig. 6 Three hydraulic cylinder force change curves

\section{Summary}

In this paper, we analysis the light hydraulic excavator working device of the kinematics and dynamics simulation by using ADMAS software powerful analysis function. We have been obtained the scope of work and the main basic parameters of excavator boom, bucket rod, bucket and the whole machine by kinematics simulation; and obtained the articulated point and the force curve of hydraulic cylinder by dynamic simulation. we also got the curve of the movement process and model simulation analysis of the operation situation of excavator working device in this paper, the conclusion can be used as the theory basis of the design and optimization of the excavator working device.

\section{Acknowledgments}

This work was supported by the Applied Basic Research Programs of Changzhou under grant number CJ20140027, the Guangxi Key Laboratory for manufacturing system and advanced manufacturing technology under Grant number 13-051-09S09 and the graduate student innovation project of Changzhou campus of HoHai university under grant number XZX/14B001-05. 


\section{References}

1. DiMaio S.P, Salcudean S.E, Reboulet C,Proc IEE

E Int Conf Rob Autom 1,52(1998)

2. Haga M, Hiroshi W, Fujishima K,Mechatronics 11, 665(2001)

3.Imanishi E, Nanjo T, Hiroka E,Nippon Kikai Gakk ai Ronbunshu C 69,2336(2003)

4. Ye Li, Xiang Xu, Qin Qiu,Proc. World Congr. I ntelligent Control Autom. WCICA 2,6915(2006)

5. XUE Caijun, QIU Qingying, FENG Peien, ICED,7 $72(2003)$

6. Liu Jing, Pan Shuang-Xia, Feng Pei-En,J ZHEJIG UNIV 38,1490(2004)

7. Weidong Zhou, Man. Auto. 7,166(2010)

8. Zhenpei Qin,Junping Zhu, Agr. Mec. Research 4, 46(2011)

9. Wei Huang,Qua. Tec. Supervision 5,27(2008)

10. Deli Kong, Keli Zhao,Hydraulic excavator(Beijing, 2007) 